\title{
18F-fluorodeoxyglucose positron emission tomog- raphy/computed tomography of bilateral primary ureter lymphoma
}

\author{
Liang Yin ${ }^{1,2,}{ }^{,}$, Yusheng Zhen ${ }^{3,}$, Zhichun $\operatorname{Lin}^{1}$, Weilong Li ${ }^{2,4}$, and Zhaowei Meng ${ }^{2}$
}

${ }^{1}$ Department of Nuclear Medicine, Pingjin Hospital, Characteristic Medical Center of Chinese People's Armed Police Forces, Tianjin; ${ }^{2}$ Department of Nuclear Medicine, Tianjin Medical University General Hospital, Tianjin; ${ }^{3}$ Division of Nuclear Medicine, Department of Radiology, Tianjin Union Medicine Center, Tianjin; ${ }^{4}$ Department of Nuclear Medicine, The Affiliated Yantai Yuhuangding Hospital of Qingdao University, Yantai, China

Received: August 4, 2020 Revised : August 18, 2020 Accepted: August 18, 2020

\section{Correspondence to Zhaowei Meng, M.D.}

Tel: +86-18622035159

Fax: +86-022-27813550

E-mail: zmeng@tmu.edu.cn https://orcid.org/0000-00024478-878X

\footnotetext{
*These authors contributed equally to this work.
}

A 44-year-old asymptomatic male was incidentally found to have bilateral hydronephrosis in a health examination. Laboratory tests revealed elevated creatinine level $(142 \mu \mathrm{mol} / \mathrm{L})$ in serum and occult blood in urine. Magnetic resonance imaging showed bilateral ureteral thickening with complete obstruction of the right ureter, stenosis of the left proximal ureter and bilateral hydronephrosis, as well as retroperitoneal adenopathy (Fig. $1 \mathrm{~A}$ and ${ }_{1 B}$ ). A routine and delayed (6o minutes after intramuscular injection of furosemide) $18 \mathrm{~F}$-fluorodeoxyglucose $\left({ }^{18} \mathrm{~F}-\mathrm{FDG}\right)$ positron emission tomography/computed tomography (PET/ $\mathrm{CT}$ ) imaging revealed multiple hypermetabolic masses and nodules in the wall of bilateral ureters and para-aortic areas (Fig. $1 \mathrm{C}-1 \mathrm{G}$ ). Considering the lesion as a malignancy, a laparoscopic needle biopsy was performed on several hypermetabolic lymph nodes, and histopathological examination revealed follicular lymphoma (Fig. 2). We also performed bone marrow biopsy which did not show lymphoma involvement. After six courses (four cycles of rituximab plus cyclophosphamide, hydroxyldaunorubicin, oncovin and prednisone [R-CHOP] and two courses of bendamustine plus rituximab) of chemotherapy, the patient showed complete resolution on a follow-up ${ }^{18}$ F-FDG PET-CT scan (Fig.1 $\mathrm{H}-1 \mathrm{~K}$ ). The patients remained disease-free 2 years after the treatments.

The definition of primary extranodal lymphoma is still a controversy. As to the present case, considering the patient had only regional lymph node involvement, and referring to the recommendation by some scholars, it was categorized as primary ureter lymphoma. However, primary lymphoma involving the ureter is exceedingly rare, most cases were unilateral, with only five cases (including the current case) of primary bilateral ureteral lymphoma being reported in the literature so far. In addition, this report also highlights the role of ${ }^{18} \mathrm{~F}$-FDG PET/CT in unexplained hydronephrosis condition and in its treatment monitoring.

All procedures performed in studies involving human participants were in accordance with the ethical standards of the institution and with the principles of the 1964 Declaration of Helsinki and its later amendments or comparable ethical standards. Informed consent was obtained from the patient for the anonymous use of patient clinical, imaging, and histological data. 

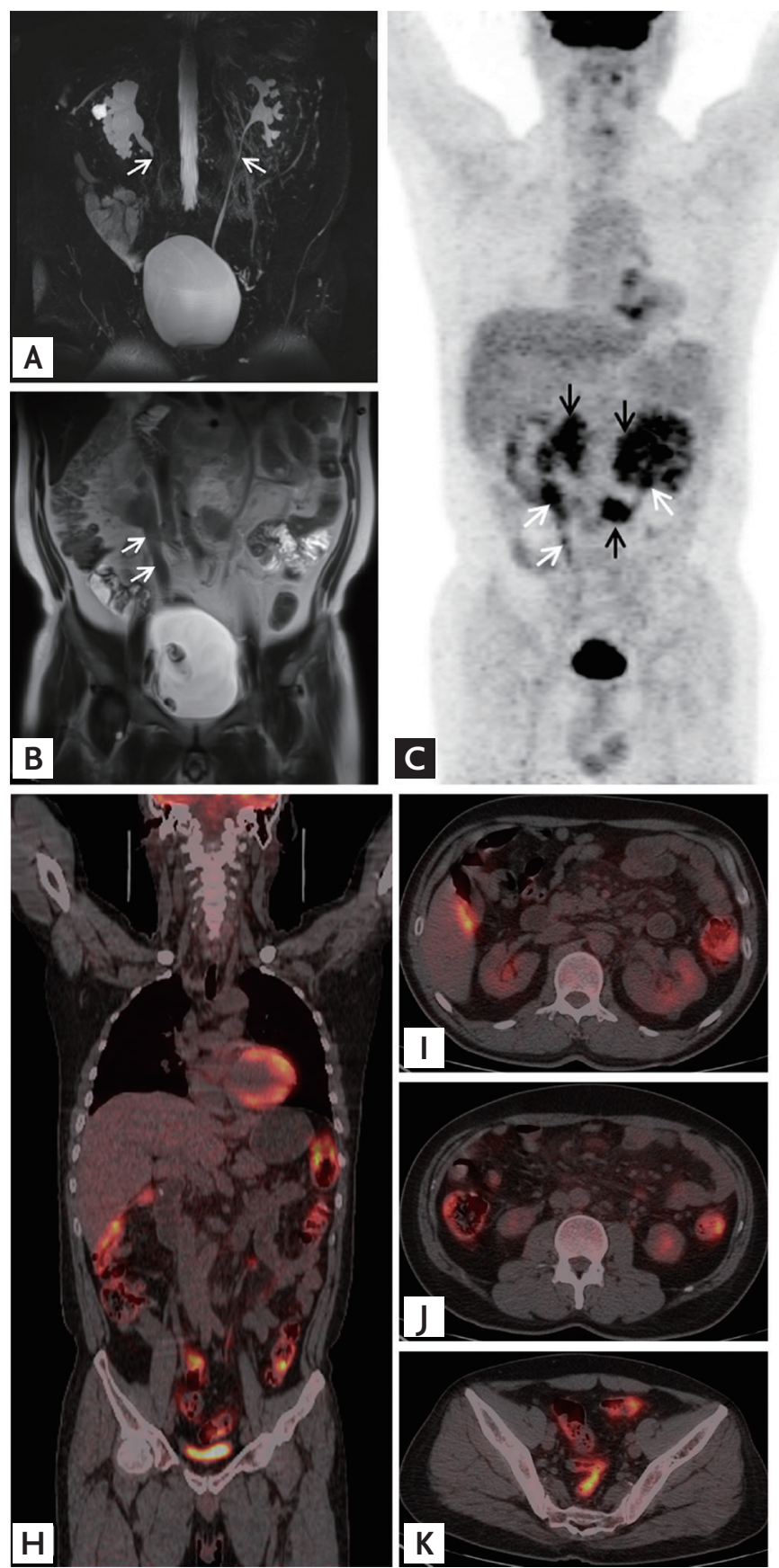
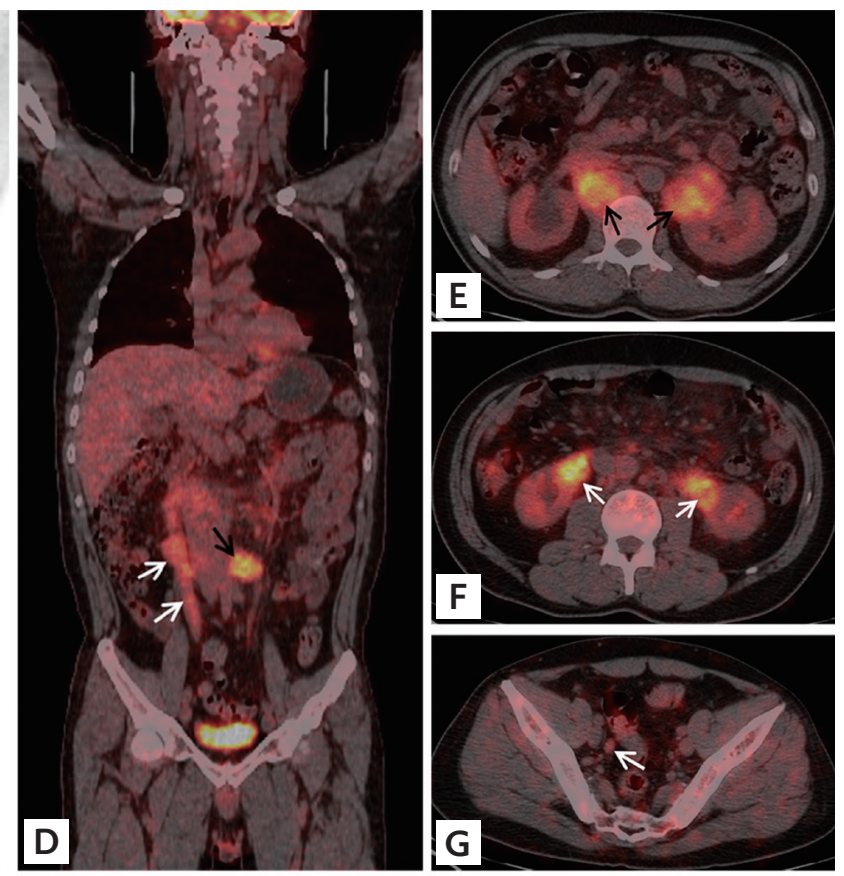

Figure 1. (A) Magnetic resonance urography showed bilateral hydronephrosis, complete obstruction of the right ureter, and stenosis of the left proximal ureter. (B) Magnetic resonance imaging (MRI) revealed the thickened upper middle section of right ureter, MRI also exhibited low intensity on T2-weighted images and retroperitoneal adenopathy. A whole body $18 \mathrm{~F}$-fluorodeoxyglucose $\left({ }^{18} \mathrm{~F}\right.$-FDG) positron emission tomography/computed tomography (PET/CT) was performed ("white arrows" in the figure showed the lesion of the ureter, and "black arrows" showed the lesion of local lymph node). (C) The maximal intensity projection image revealed multiple hypermetabolic masses and nodules in the wall of bilateral ureters and para-aortic areas. (D) The coronal fused ${ }^{18}$ F-FDG $\mathrm{PET} / \mathrm{CT}$ image showed a zonal thickening and hypermetabolic of the right ureter, especially in the middle and upper segments, ${ }^{18}$ F-FDG PET/CT also indicated retroperitoneal lymphadenopathy. In the axial fused images, retroperitoneal lymphadenopathy and bilaterally hydronephrosis were demonstrated (E), and thickening of the bilaterally ureters were shown as well (F, G). Complete resolution after the treatments was demonstrated on a follow-up ${ }^{18}$ F-FDG PET$\mathrm{CT} \operatorname{scan}(\mathrm{H}, \mathrm{I}, \mathrm{J}, \mathrm{K})$. 

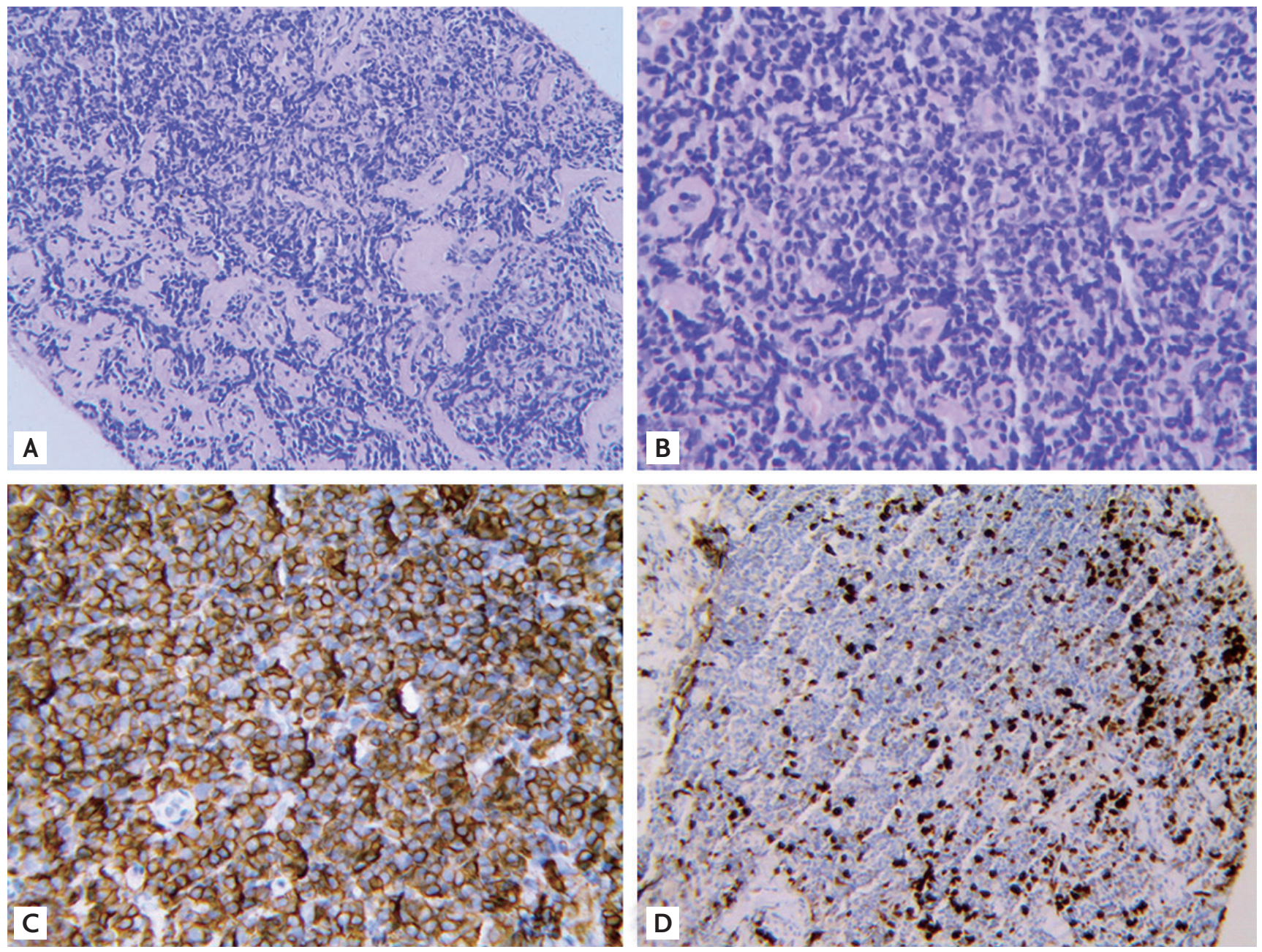

Figure 2. (A, B) H\&E staining exhibited atypical lymphocytes from follicular nodules (A: $\times 100 ; \mathrm{B}: \times 200)$. Immunohistochemical staining demonstrated a positive expression of (C) CD20 ( $\times 200)$, and (D) Ki- 67 index was $10 \%$ approximately $(\times 200)$.

\section{Conflict of interest}

No potential conflict of interest relevant to this article was reported. 